sumption that they are actual ellipsoids of equilibrium. Our best guide is $G$. $H$. Darwin's work on masses of homogeneous, incompressible fluid. The actual stars are undoubtedly denser toward the center, which tends toward a smaller ellipticity, and they are composed of elastic and expansible material, which, as Feans has shown ${ }^{1}$ ), may cause ellipticity or even instability to set in for a much smaller rate of rotation than would be the case for a mass of incompressible fluid of equal mean density. The results given below may perhaps give a fair approximation to the relative magnitude of the third axis of the ellipsoids, but should be considered as only an indication of the possible order of magnitude of their densities.

From Darwin's paper ${ }^{2}$ ) we may take the following values for the dimensions and angular velocity of the Facobian ellipsoids of homogeneous incompressible fluid, the unit of length being the radius of the sphere of equal volume, and the unit of density being so chosen that the Gaußian constant is unity. The fitth of these computed ellipsoids represents the condition just beyond which instability occurs and the pear-shaped figure begins to form.

Table III. Facobi's Ellipsoids of Equilibrium.

\begin{tabular}{c|c|c|c|c|c|c}
\hline No. & $a$ & $b$ & $c$ & $\omega^{2} / 4 \pi \varrho$ & $b / a$ & $c / a$ \\
\hline \hline 1 & 1.197 & 1.197 & 0.698 & 0.0936 & 1.000 & 0.583 \\
2 & 1.279 & 1.123 & 0.696 & 0.093 & 0.879 & 0.544 \\
3 & 1.383 & 1.045 & 0.692 & 0.0906 & 0.756 & 0.501 \\
4 & 1.601 & 0.924 & 0.677 & 0.0830 & 0.577 & 0.423 \\
5 & 1.899 & 0.811 & 0.649 & 0.0705 & 0.427 & 0.342 \\
6 & 2.346 & 0.702 & 0.607 & 0.0536 & 0.299 & 0.259 \\
7 & 3.136 & 0.586 & 0.545 & 0.0334 & 0.187 & 0.174
\end{tabular}

\title{
Die Veränderlichkeit des Polarsterns. Von Ant. Pannekoek.
}

Als ich in den Jahren 1889 und 1890 Beobachtungen der Helligkeit mehrerer Sterne zweiter und dritter Größe nach Argelanders Methode anstellte, zeigte sich bei einigen Sternen ein starker Verdacht der Veränderlichkeit, u. a. bei $\boldsymbol{\alpha}$ Ursae minoris. Die Schätzungen zeigten eine Periode von etwas weniger als 4 Tagen; da aber die Amplitude äußerst gering war, gelang es mir nicht, einen Wert für die Periode zu finden, der den Beobachtungen genligte, und damit die Veränderlichkeit zweifelsfrei festzustellen. Als dann nachher Camphell eine Veränderlichkeit der visuellen Geschwindigkeit in einer Periode von 3.968 Tagen feststellte, und sich bei einer Untersuchung im Jahre r 906 zeigte, daß der Polarstern den $c$-Charakter des Spektrums und die geringe Dichtigkeit mit den kurzperiodischen Veränderlichen gemeinsam hat, wurde die Realität einer Veränderlichkeit im hohen Maße wahrscheinlich. Hertzsprung hat dann neulich auf photographischem Wege eine Veränderlichkeit mit einer Amplitude von 0.17 Größenklassen nachgewiesen und Stebbins hat sie mit dem Selenphotometer bestätigt.

Die Beobachtungen, die ich in den Jahren 1890 bis I 899 mit freiem Auge angestellt habe, zerfallen in zwei getrennte Reihen, Vergleichungen mit $\alpha$ Persei, $\beta$ und $\gamma$ Andromedae in der zweiten und Vergleichungen mit $\epsilon$ und $\eta$ Ursae majoris in der ersten Hälfte jedes Jahres. Werden sie alle
In Table III the axes of the ellipsoids are designated by $a, b, c$, in descending order of magnitude; the angular velocity is denoted by $\omega$, and the density by $\varrho$. For the three ellipsoidal stars, the quantity $b / a=\left(\mathrm{r}-\varepsilon^{2}\right)^{1 / 2}$ would be obtained from the light curves if the inclination were known. We will take $i=90^{\circ}$, which is probably near the truth, at least for the two stars of greatest range, and this will give the minimum value of the ellipticity. Plotting the values of $b / a$ in the above table against $c / a$, and against $\omega^{2} / 4 \pi \rho$, we can read from the curves the values of the angular velocity and relative polar axis for each of the variables. The density is then readily computed from the known period of rotation. The results are tabulated below.

\begin{tabular}{|c|c|c|c|c|c|c|}
\hline & \multicolumn{2}{|c|}{ RU Camelopardalis } & \multicolumn{2}{|c|}{ SZ Tauri } & \multicolumn{2}{|c|}{ S Antliae } \\
\hline & Uniform & Darkened & Uniform & Darken. & Uniform & Darken. \\
\hline$\varepsilon^{2}$ & 0.82 & 0.62 & 0.67 & 0.47 & 0.475 & 0.33 \\
\hline$b / a$ & 0.424 & 0.617 & 0.574 & 0.728 & 0.724 & 0.819 \\
\hline$c / a$ & 0.341 & 0.443 & $0.42 \mathrm{I}$ & 0.490 & 0.489 & 0.524 \\
\hline$\omega^{2} / 4 \pi \varrho$ & 0.0703 & 0.0853 & 0.0828 & 0.0897 & 0.0897 & 0.0922 \\
\hline 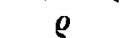 & 0.00003 & 0.00003 & 0.0014 & $0.00 I_{3}$ & o.I I 9 & 0.116 \\
\hline
\end{tabular}

The densities are expressed in terms of the mean solar density. It should be noted that the uniform solution for RU Camelopardalis gives dimensions practically identical to those of the homogeneous ellipsoid that is near to instability and to the transformation into the pear-shaped figure.

Princeton University Observatory, I 9 I 3 March I. Harlow Shapley.

1) Philosophical Transactions, A, 201.157; A, 199.r.

2) Roy. Soc. Proc, 41.319, 1887 .

mit dem Periodenwert 3.968 auf eine einzige Periode, 3 . bis 7. August 1894, zurückgebracht und dann nach der Phase geordnet, so ergeben sich folgende Mittelwerte:

Erste Reihe.

Vergl.-St. : $\alpha$ Persei $=6.3$, $\beta$ Andr. $=3.8, \gamma$ Andr. $=3 . \mathrm{I}$. Epoche Helligk. Ánz. $B-\mathrm{R}$

Aug. 3.12 3.72 (18) +0.03 $3.423 .94(16)+0.11$

3.734 .1 I $(2 I)+0.08$

$\begin{array}{lllll}3.96 & 3.91 & (17) & -0.29\end{array}$

$\begin{array}{llll}4.22 & 3.94 & (20) \quad-0.42\end{array}$

4.544 .68 (I 7$)+0.18$

$4.764 .86(16)+0.33$

4.944 .76 (16) +0.24

5.184 .1 I (I 7$)-0.34$

5.524 .45 (14) +o.19

5.76 4.I I $(20)+0.02$

$\begin{array}{lllll}5.94 & 3.67 & (\mathrm{x} 8) & -0.29\end{array}$

$\begin{array}{lllll}6.23 & 3.67 & (16) & -0.11\end{array}$

6.463 .78 (I 4$)+0.10$

6.733 .79 (I9) +0.16

Die Veränderlichkeit des Polarsterns tritt in beiden

\section{Zweite Reihe.}

Vergl.-St. : $\varepsilon$ Ursae maj. $=2.4$, $\eta$ Ursae maj. $=0.0$.

Epoche Helligk. Anz. $B-\mathrm{R}$

$\begin{array}{lllll}\text { Aug. 3.2 I } & 0.59 & \text { (I } 8) & -0.03\end{array}$

$\begin{array}{lllll}3.48 & 0.68 & \text { ( } 16) & -0.08\end{array}$

$\begin{array}{lllll}3.65 & 0.59 & \left(I_{3}\right) & --0.28\end{array}$

3.92 I.13 (18) +0.07

$4.251 .39(20)+0.10$

$4.46 \quad 1.40(22) \quad 0.00$

$4.651 .69(24)+0.22$

$4.941 .55(14)+0.05$

$5.221 .20(\mathrm{I} 7)-0.23$

$5.46 \quad 1.27(18)-0.03$

$5.721 .02(20)-0.11$

6.220 .74 (19) -0.04

$\begin{array}{llll}6.48 & 0.86(16)+0.22\end{array}$

$\begin{array}{llll}6.86 & 0.67 & \text { (1 } 6) & \text { +o. I I }\end{array}$ 
Reihen klar zu Tage. Sucht man die Helligkeit durch eine Sinusoide darzustellen, so findet man

I. Reihe: $4.08+0.45 \sin (\varphi-72: 0)$ Max. Aug. 4.79 $\pm 00_{\text {I I }}$ 2. Reihe: $1.03+0.47 \sin \left(\varphi-78^{\circ} .9\right) \quad$ " $4.86 \pm 0^{\mathrm{d}} \mathrm{x} \mathrm{r}$.

Der $m$. F. einer Mittelzahl ergibt sich zu 0.22 , einer einzigen Beobachtung $\mathrm{zu} 0.84$, und die Zeit des Maximums wird, nach Reduktion auf Greenwich

I 894 Aug. $4.8 \mathrm{I}=$ J. D. 24 r $3045.8 \mathrm{I} \pm 0.08 \mathrm{~m}$. Z. Gr.

Zur Reduktion der benutzten Skalen auf photometrische Größen wurde für die Vergleichsterne und einige weitere daran angeschlossene Sterne die Größe berechnet, und dafür das Mittel der Ergebnisse von Harv. Ann. I 4, Harv. Ann. 44, Potsdam Photometer CII und Potsdam Photometer CIII genommen, nachdem sie auf das in meiner Dissertation aufgestellte System $H_{r}$ reduziert waren ${ }^{1}$ ).

\begin{tabular}{|c|c|c|c|c|c|c|c|}
\hline a Pers & $\begin{array}{l}\text { Größe } \\
1 \mathrm{~m} \cdot 91\end{array}$ & $\begin{array}{l}\text { Farb } \\
3 \cdot 4\end{array}$ & $\begin{array}{r}\text { Skale! } \\
6^{n} \cdot 3\end{array}$ & $\varepsilon$ Ursae maj. & $\begin{array}{l}\text { Größe } \\
\mathrm{I} \cdot 89\end{array}$ & $\begin{array}{l}\text { Farbe } \\
1: 8\end{array}$ & $\begin{array}{r}\text { Ska } \\
2 !\end{array}$ \\
\hline$\alpha$ Arietis & 2.03 & $5 \cdot 4$ & $5 \cdot 4$ & $\eta \quad$ & 2.02 & 1.4 & $\circ$ \\
\hline$\beta$ Andromed. & 2.10 & 6.2 & 3.8 & 5 & 2.20 & 2.1 & \\
\hline$»$ & 2.14 & 5.2 & 3.1 & $\alpha$ Coron. bor. & $2 \cdot 37$ & 1.8 & -4.8 \\
\hline$\triangleright$ & 2.17 & I. 8 & $2 \cdot 3$ & $\varepsilon$ Bootis & $2.5^{\circ}$ & 4.8 & $-5 \cdot 7$ \\
\hline$\gamma$ Cassiopeiae & 2.28 & $2 . I$ & 0.8 & $\beta$ Ursae maj. & 2.54 & I. 7 & -8 \\
\hline$\beta \quad "$ & 2.41 & 2.9 & -1.7 & $\gamma \quad »$ & 2.55 & I. 8 & -9 \\
\hline$\alpha$ Ursae maj. & I. 85 & 4.9 & 4.0 & & & & \\
\hline
\end{tabular}

Die Ausgleichung mit Hinzuziehung eines von der Farbe abhängigen Einflusses ergab

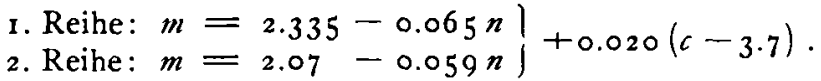

Die Darstellung der Helligkeit des Polarsterns durch eine Sinusformel wird dann in Größenklassen

$$
\begin{aligned}
& \text { 1. Reihe: } 2.07-0^{\mathrm{m}} 029 \sin (\varphi-72.0) \\
& \text { 2. Reihe: } 2.01-0^{\mathrm{m}} 028 \sin \left(\varphi-78^{\circ} .9\right) \text {. }
\end{aligned}
$$

Die Amplitude des Lichtwechsels ist also 0.057 Größenklassen.
In ähnlicher Weise habe ich die Beobachtungen untersucht, die $G$. Müller ${ }_{1878-8} \mathrm{I}$ in Potsdam zur Bestimmung der atmosphärischen Extinktion anstellte ${ }^{2}$ ) und die aus photometrischen Messungen der Helligkeitsdifferenzen zwischen $\boldsymbol{\alpha}$ Ursae min. und 5 anderen Sternen bestehen. Nach Ausschluß aller als unsicher bezeichneten und aller Beobachtungen, bei denen $z>60^{\circ}$, wurden für die übrigen die "Abweichungen vom Mittel " (Tabelle IV vorletzte Spalte S. 251-265) mit umgekehrtem Zeichen (damit eine größere Helligkeit des Polarsterns durch positive Abweichungen bezeichnet wird) nach der Phase geordnet, zu Mittelwerten zusammengenommen, und mit dem Faktor eine Einheit $=0.0025$ multipliziert. Diese Mittel sind:

Phase 1879 Abweich. B-R $\mid$ Phase 1879 Abweich. $B-R$

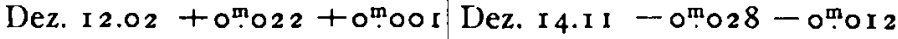

$12.34+0.030 \quad 0.000 \quad 14.50-0.009+0.015$

$12.64+0.047+0.014+14.81-0.009+0.013$

$12.92+0.008-0.021 \quad 15.05-0.021-0.004$

$13.21+0.028+0.008+15.20-0.048-0.036$

I $3.63+0.006+0.003 \quad$ I $5.34+0.017+0.022$

I $3.84-0.010-0.004 \quad 15.69+0.008-0.001$

Sie lassen sich durch eine Sinusformel darstellen:

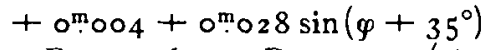

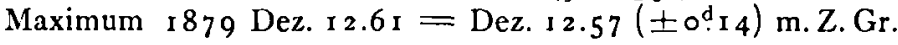

Für die nahezu gleichzeitigen photometr. Messungen auf der Harvard Sternwarte, bei denen Polaris als Vergleichstern benutzt wurde, hat Pickering schon die Mittelwerte der Abweichungen zusammengestellt ${ }^{3}$ ). Sie lassen sich durch die Sinusformel $-\circ^{\mathrm{m}} \circ \mathrm{I} \mathrm{I}+0^{\mathrm{m}} \circ 39 \sin \left(\varphi+254^{\circ}\right)$ am besten darstellen und ergeben ein Maximum für Phase 2.16, also für J. D. $2408228.45 \pm 0^{d} 24$ und eine Amplitude von 0.078 Größenklassen.

Die folgende Zusammenstellung gibt die Resultate der bisher bearbeiteten Beobachtungsreihen, nebst einer Ver-

\begin{tabular}{|c|c|c|c|c|c|c|}
\hline Jahr & $E$ & Maximum & $B-R$ & Amplitude & Beobachter & $\mathrm{B}-\mathbf{R}^{\prime}$ \\
\hline I 879 & -2845 & $2407696.57 \pm 0^{d} 14$ & -0.05 & $0^{\mathrm{m}} .056$ vis. & Miiller & $-0^{\mathrm{d}} \mathbf{1}_{3}$ \\
\hline I 88 I & -27 I & $8228.45 \pm 0.24$ & +0.11 & 0.078 vis. & Harvard & +0.03 \\
\hline I 894 & -1497 & $2413045.81 \pm 0.08$ & +0.20 & 0.057 vis. & Pannekoek & +0.12 \\
\hline I910 & $\circ$ & $8985.86 \pm 0.08$ & 0.00 & $0.17 \times$. photogr. & Hertzsprung & -0.07 \\
\hline 9 I I & $\circ$ & $8985.94 \pm 0.09$ & +0.08 & 0.078 selen. & Stebbins & +0.01 \\
\hline
\end{tabular}
gleichung mit der Hertzsprungschen Formel:

$$
\text { Max. }=\text { J. D. } 24 \text { I8985.86+3 } 4968 \text { × } E \text {. }
$$

Der Versuch, aus diesen Abweichungen eine Korrektion der Formel herzuleiten, ergab für die Periode nur eine Änderung - od.00001 \pm 0 d.00004, und für die Epoche eine Korrektion +odo7, wobei die Abweichungen der letzten Spalte übrigbleiben. Es ist also nach dem vorhandenen Material anzunehmen:

$$
\text { Maximum J. D. } 24 \text { I } 8985.93( \pm 0 \text { d.06) }+3 \text { d.96809 } E( \pm 0 \text { d.00004 }) \text {. }
$$

Bremen, I 9 I 3 Febr. I.

Ant. Pannekoek.

1) A. Pannekoek. Untersuchungen über den Lichtwechsel Algols. S. 158. Dort sind die Korrektionen der Katalogwerte für Harv. 14 und Potsdam CII gegeben. Fur Potsdam CIII ist eine konstante Korrektion $-0^{\text {n. }} \cdot 23$ angenommen; für Harv. 44 sind zuerst die Differenzen $H_{44}-H_{14}$ angebracht, die aus den in Potsdam Publ. Bd. 17, p.XXIII gegebenen Zahlen durch Ausgleichung gewonnen wurden.

2) Publikationen des Astrophysikalischen Observatoriums zu Potsdam Bd. III.

3) Astron. Nachr. 4597 (Bd. 192, S. 219); Harv. Zirk. 174, wo die Berechnung der Epoche des Maximums verfehlt ist $(E=2073$ ergibt die Formel 2408226.29, nicht 2408227.0, wie dort angenommen wurde). 